\title{
CHANGES IN NOVEL GASTROINTESTINAL AND RENAL INJURY MARKERS IN THE BLOOD PLASMA OF SHEEP FOLLOWING INCREASING INTRAVENOUS DOSES OF TOLFENAMIC ACID
}

\author{
Ramazan YILDIZ ${ }^{1 *}$, Orhan CoRUM ${ }^{2}$, Orkun ATIK ${ }^{3}$, Duygu Durna CoRUM ${ }^{2}$, Feray ALtAN ${ }^{5}$, \\ Mahmut $\mathrm{OK}^{5}$ and Kamil UNEY ${ }^{6}$ \\ ${ }^{1}$ Department of Internal Medicine, Faculty of Veterinary Medicine, University of \\ Mehmet Akif Ersoy, 15030, Burdur, Turkey; ${ }^{2}$ Department of Pharmacology and \\ Toxicology, Faculty of Veterinary Medicine, University of Kastamonu, Kastamonu, \\ Turkey; ${ }^{3}$ Department of Pharmacology and Toxicology, Faculty of Veterinary Medicine, \\ University of Afyon Kocatepe, Afyonkarahisar, Turkey; ${ }^{4}$ Department of Pharmacology \\ and Toxicology, Faculty of Veterinary Medicine, University of Dicle, Diyarbakır, \\ Turkey; ${ }^{5}$ Department of Internal Medicine and ${ }^{6}$ Department of Pharmacology and \\ Toxicology, Faculty of Veterinary Medicine, University of Selcuk, Konya, Turkey
}

(Received 23 June 2018; accepted 15 February 2019)

The administration of high doses of non-steroidal anti-inflammatory drugs (NSAID), such as tolfenamic acid (TA), has undesirable effects on different organs. Some novel biomarkers have been reported that can determine the gastrointestinal and renal injury caused by a high dose of NSAIDs or other toxic substances. This study was aimed at determining the changes in gastrointestinal (TFF2 and HYP), renal (NGAL and KIM-1) and cardiac (cTn-I, CK-MB) injury markers after the use of increasing intravenous doses of TA in sheep. TA was administered intravenously to groups of six sheep each, at the dose levels of 0 (Group 0, i.e., G0), 2 (G2), 4 (G4), 8 (G8) and 16 (G16) mg/kg. The concentrations of the studied biomarkers were measured at $3,9,18$ and $36 \mathrm{~h}$ after administration of TA. The TFF2 and NGAL concentrations in G16 were found to be significantly higher $(\mathrm{P}<0.05)$ than in the other groups except for $\mathrm{G} 8$ at different sampling times. HYP concentration in G16 was observed to be significantly $(\mathrm{P}<$ $0.05)$ lower than that in all other groups at $36 \mathrm{~h}$. KIM-1 level in G16 was significantly $(\mathrm{P}<0.05)$ higher than in all other groups at different sampling times. An increase in the renal markers, KIM-1 and NGAL, in G8 was observed before any change in plasma creatinine and urea. The cardiac marker cTn-I in G16 was significantly $(\mathrm{P}<0.05)$ higher than in other groups at different sampling times. The results showed that the novel biomarkers (HYP, TFF2, NGAL, and KIM-1) can be used to determine gastric and renal injury in sheep. acid

Key words: Biomarkers, cardiac, gastrointestinal, injury, renal, tolfenamic

\footnotetext{
*Corresponding author; E-mail: ramazanyildiz@mehmetakif.edu.tr;
} Phone: 0090 (248) 213-2207; Fax: 0090 (248) 213-2001 
Non-steroidal anti-inflammatory drugs (NSAIDs), including tolfenamic acid (TA), are one of the most commonly used classes of drugs in humans and animals. NSAIDs have analgesic, antipyretic, and anti-inflammatory properties. TA has been approved for use in mastitis, metritis, and respiratory system infections in cattle and for musculoskeletal disorders and the reduction of postoperative pain in dogs and cats (CVMP, 1997). NSAIDs are associated with adverse effects like renal failure, gastric ulcers, and bleeding disorders after injuries and/or surgical interventions (Curry et al., 2005). There are classic/conventional and novel biomarkers to determine the gastrointestinal, renal, and cardiac injuries. The increasing concentration in trefoil factor 2 (TFF2), which is a peptide (Poulsen et al., 1999) and the decreasing concentration in hydroxyproline (HYP), which is an amino acid (Takeuchi et al., 2014), have been used to determine the gastrointestinal damage. TFF2 is expressed and secreted preferentially by gastric mucous neck cells (Zhang et al., 2016) and plays a key role in the maintenance of mucosal integrity (Kaise et al., 2013). HYP is a characteristic amino acid of collagen and is mostly used as an indicator to determine the amount of collagen (Zhang et al., 2016) in gastric (Takeuchi et al., 2013) and lung tissue (Shao et al., 2015). Decreased concentrations of HYP in stomach tissue extract and serum have been reported in NSAID-induced gastric ulcers (Takeuchi et al., 2013). It has been reported that these changes are due to the activation of collagenase by NSAID-induced mitochondrial depression and by lesions present in the stomach. There is a significant correlation between the changes in HYP levels in the stomach and serum (Takeuchi et al., 2014).

Early diagnosis of NSAID-induced renal injury is important but difficult. In clinical settings, the follow-up of patients is mainly based on the detection of serum creatinine $(\mathrm{Cr})$ and blood urea, and on urinalysis (Fuchs and Hewitt, 2011). Owing to the poor sensitivity and specificity of these traditional markers, new indicators are required for a more accurate and early detection of kidney damage associated with the side effects of NSAIDs (Sistare et al., 2010). In renal tissue injuries, many protein products such as neutrophil gelatinase-associated lipocalin (NGAL) and kidney injury molecule-1 (KIM-1) appear in the blood and may serve as new biomarkers for the early detection of acute kidney injury (AKI) (Ozer et al., 2010; Luo et al., 2016). NGAL, a small protein from the lipocalin family, is expressed exclusively in the tubular epithelium of the distal nephron and is released by tubular epithelial cells during AKI (Zhao et al., 2010). NGAL was used to determine the risk of AKI in dogs (Diniz et al., 2010). KIM-1 may facilitate accurate prediction of nephrotoxicity in the preclinical stages of drug screening (Vaidya et al., 2010).

The US Food and Drug Administration has mandated a 'black box' warning for all NSAIDs to describe the potential risk of serious cardiovascular events (Hermann, 2009). Traditional cardiac biomarkers like troponin-I (cTn-I) and 
Creatine Kinase-MB fraction (CK-MB) can be used to detect cardiac injury in animals (Undhad et al., 2012).

The aim of this study was to establish the superiority of novel gastric (TFF2 and HYP) and renal (NGAL and KIM-1) tissue damage biomarkers over the conventional renal function markers ( $\mathrm{Cr}$ and urea) and heart tissue damage markers (cTn-I and CK-MB) in the blood plasma of sheep in an experimental model where tissue damage was induced by increasing doses of TA $(2,4,8$ and $16 \mathrm{mg} / \mathrm{kg})$.

\section{Materials and methods}

\section{Animals}

Thirty clinically healthy Akkaraman female sheep weighing between 38 and $45 \mathrm{~kg}$ and aged 2-3 years were selected for the study. The animals were checked clinically and haematologically before and after the one-week adaptation period. The healthy-looking animals were subjected to a check-up profile [creatinine, urea, alkaline phosphatase (ALP), alanine aminotransferase (ALT), aspartate aminotransferase (AST), gamma glutamyltransferase (GGT) and total protein (Table 2)] and those with normal values were randomly assigned to five equal groups (G0, G2, G4, G8, and G16). During the experimental period, all sheep were kept together, outdoors during the day and housed at night. The animals were fed daily on a ryegrass and clover hay mix along with a supplementary concentrate. Water was given ad libitum. The experimental procedures were approved by the Ethics Committee of the Mehmet Akif Ersoy University (2017-280).

\section{Experimental design}

In the study, the animals were randomly assigned to five equal groups. Tolfenamic acid (Tolfine, Novakim, Kocaeli, Turkey) was administered intravenously to each group at single dose levels of 2 (G2), 4 (G4), 8 (G8), and 16 (G16) $\mathrm{mg} / \mathrm{kg}$. The control group (G0) received only sterile physiological saline solution in a similar manner. The blood samples were collected into tubes containing heparin at $3,9,18$, and $36 \mathrm{~h}$ post-dosing, via a catheter placed in the right jugular vein. Blood samples were centrifuged $(10 \mathrm{~min}$ at $2500 \times \mathrm{g})$ and the plasma was stored at $-70{ }^{\circ} \mathrm{C}$ until assayed.

\section{Laboratory analysis}

The concentrations of gastrointestinal (TFF2 and HYP) and renal (NGAL and KIM-1) markers in the plasma were determined using commercially available sheep specific ELISA kits (MyBiosource, San Diego, USA) following the manufacturer's recommendation. Absorption was determined using a microplate reader (ELX800 universal microplate reader, BIOTEK ${ }^{\circledR}$, USA). The validation data provided by the manufacturer of the ELISA kits were used in the study. For 


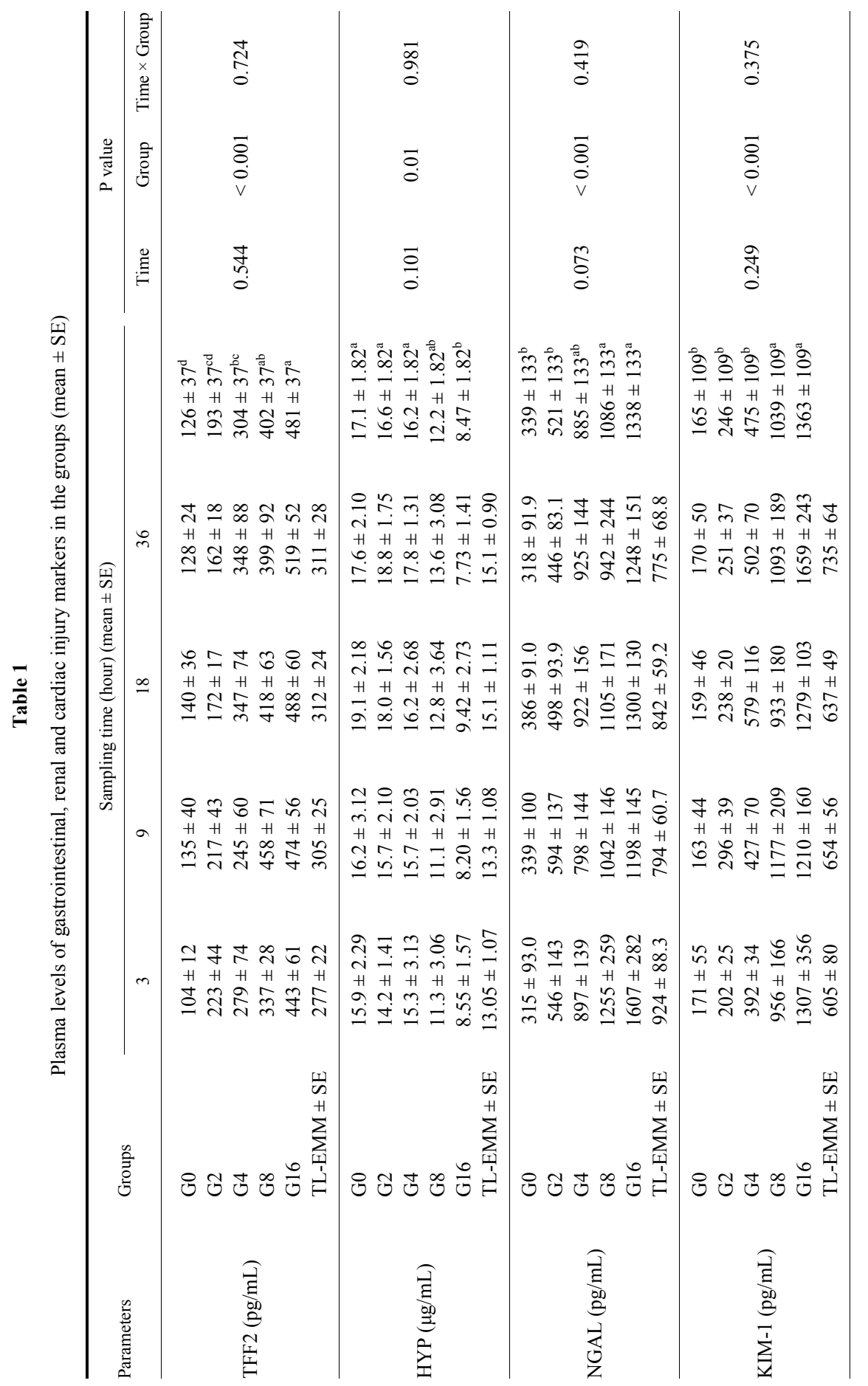




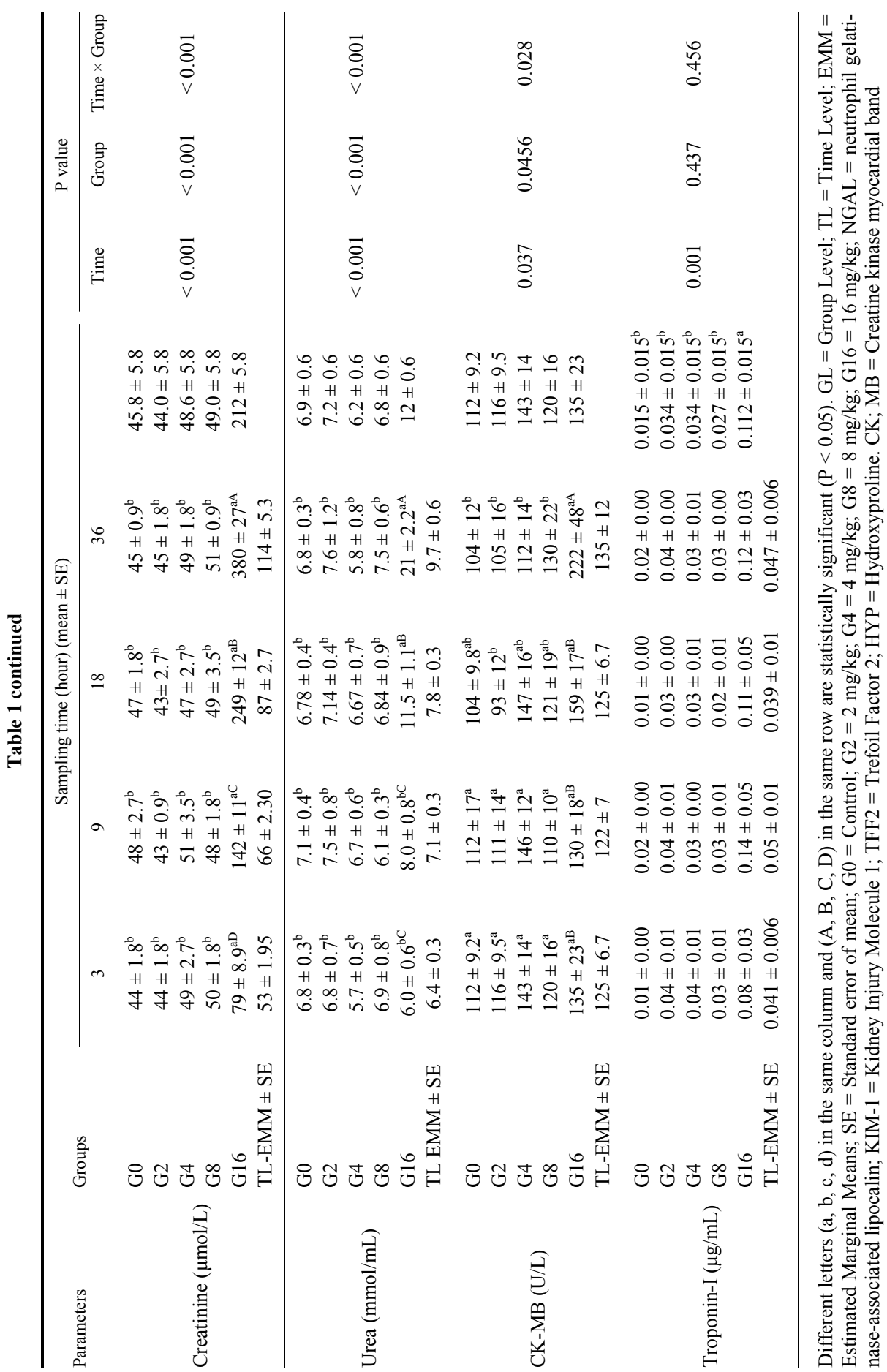


NGAL and KIM-1, the intra-assay and inter-assay coefficients of variation (CV) were $<10 \%$ and the minimum detectable concentration (MDC) was $18.75 \mathrm{pg} / \mathrm{mL}$. For TFF2, the intra-assay and inter-assay CV were $<10 \%$ and MDC was $15.625 \mathrm{pg} / \mathrm{mL}$. For HYP, the intra-assay and inter-assay CV were $\leq 15 \%$ and MDC was $0.1 \mu \mathrm{g} / \mathrm{mL}$. For the detection of heart damage markers (CK-MB, cTn-I) Siemens Advia Centaur XP (Erlangen, Germany) was used. Cr and BUN were measured with commercial kits using an autoanalyzer (ILab-300 plus, Instrumentation Laboratory, Milano, Italy).

\section{Statistical analysis}

Descriptive statistics for each variable were calculated and presented as 'Mean \pm Standard Error of Mean'. Data were subjected to two-way mixed design analysis of variance (ANOVA) using a General Linear Model for repeated measures procedure to examine the effect of 'group' and 'time' for the measurements obtained. The model included 'group' and 'time' as the main effect terms and 'group $\times$ time' as the interaction effect term. In cases where Mauchly's test revealed that the assumption of sphericity was violated, the Greenhouse-Geisser adjustment was applied. Post-hoc testing for significant interactions was performed using the simple effect analysis with the Bonferroni adjustment. In cases where the interaction terms were not statistically significant, contrasts were used to analyse the main effects. A probability value of less than 0.05 was considered significant, unless otherwise mentioned. SPSS 14.01 was used for all statistical analyses.

\section{Results}

The concentrations of biomarkers (TFF2, HYP, NGAL, KIM-1, Cr, urea, CK-MB and cTn-I) are shown in Table 1 . The statistically $(\mathrm{P}<0.05)$ highest concentrations of TFF2, NGAL, KIM-1, CK-MB, cTnI and lowest concentrations of HYP were detected in G16 (Table 1).

A substantial increase in TFF2 was found in G16 (282\%) and G8 (219\%) compared to G0, while the increase in TFF2 was less severe in G4 (141\%) and G2 (53\%). On the other hand, a 50\% decrease in HYP was found in G16 compared to that obtained in G0, whereas this decrease was less severe in G8 (29\%), G4 (5\%) and G2 (3\%).

Compared with G0, the concentrations of NGAL and KIM-1 were significantly increased in G16 (295 and 726\%, respectively) and G8 (220 and 530\%, respectively), while their concentrations were found to be lower in G4 (161 and $188 \%$, respectively) and G2 (54 and 49\%, respectively). In a manner similar to the elevation in NGAL (295\%) and KIM-1 (726\%) concentrations, the levels of creatinine (363\%) were increased in G16 at the same time. The increases in renal parameters including NGAL (220\%) and KIM-1 (530\%) were more evident in G8, but this increase was just $5 \%$ in a conventional parameter such as creatinine. 


\section{Discussion}

It has been shown that novel biomarkers such as HYP, TFF2, NGAL, and KIM-1 can be used to determine the severity of gastric and renal injury in sheep. The alterations in the concentrations of HYP, TFF2, NGAL, KIM-1, Cr, BUN, cTn-I, and CK-MB in the blood plasma showed that TA at a dose of $16 \mathrm{mg} / \mathrm{kg}$ caused gastrointestinal, renal, and cardiac damages in sheep. In this study, the high doses $(8$ and $16 \mathrm{mg} / \mathrm{kg}$ ) were used only to describe the changes in the investigated parameters, not for the confirmation of the well-known dose of 2 (and 4) $\mathrm{mg} / \mathrm{kg}$ in sheep.

Indomethacin, another NSAID, has been shown to significantly increase gastric ulceration in mice deficient in TFF2 as compared to that in wild-type mice (Farrel et al., 2002). The mRNA expression of TFF2 has been found to be increased within a few minutes of induction of ulcers in rats in an experimental gastric ulcer model (Alison et al., 1995). In the present study, TFF2 in G16 was found to be statistically higher $(\mathrm{P}<0.05)$ than in G0, G2, and G4 after 3 and $9 \mathrm{~h}$ and in G0 and G2 after 18 and $36 \mathrm{~h}$. We found that TFF2 concentration in the blood plasma in other TA dose groups, except for G2, increased from after $3 \mathrm{~h}$ $(\mathrm{P}<0.05$, Table 1). An increase in TFF2 was found in G16 $(282 \%)$ and G8 (219\%) compared to G0, while the increase in TFF2 was less severe in the other groups. The major mechanism of NSAIDs in causing gastro-duodenal ulceration and bleeding is the suppression of PG synthesis. Following the inhibition of PG synthesis, gastrointestinal tissues become more sensitive to the damage caused by gastric acid. Due to the inhibition of PGs, the restoration of structure and function of the mucosa may decline after the damage (Wallace, 2008). The role of TFF2 in mucosal healing and repair is consistent with its ability to inhibit gastric acid secretion. After the gastric mucosal injury, TFF2 expression increases rapidly, which is likely to cause a reduction in the production of gastric acid (Farrel et al., 2002). HYP is another novel biomarker that can detect gastric damage associated with NSAIDs. It has been reported that the metabolic profiles of serum and gastric tissue extracts of rats show a decrease in HYP concentrations after treatment with NSAIDs (Takeuchi et al., 2013). In addition, a significant correlation was observed between the decrease in proline and HYP concentrations in serum and gastric tissue extracts. HYP has been demonstrated to be a new, non-invasive biomarker for the monitoring of NSAID-induced gastric damage and a promising alternative of the current invasive endoscopic procedures. The decrease in proline and HYP concentrations reflects an increased collagenase activity following a decrease in the collagen level of the stomach tissue (Takeuchi et al., 2013). A previous study (Takeuchi et al., 2014) also reported that HYP is a serum biomarker, which can predict NSAID-induced gastric damage in rats. In the present study, HYP level in G16 was found to be lower than that in other groups after $3 \mathrm{~h}$. Further, it was statistically lower than that in G0 af- 
ter 9 and $18 \mathrm{~h}$ or that in $\mathrm{G} 2$ after $18 \mathrm{~h}$ and was lower than in all other groups after $36 \mathrm{~h}$. A decrease in HYP was found in G16 (50\%) compared to that obtained in G0, whereas this decrease was less severe in the other groups. The decrease in HYP level seen in G16 may be associated with a decrease in the amount of collagen due to the gastric damage caused by TA administered at a dose of $16 \mathrm{mg} / \mathrm{kg}$. The percentage of the increase and decrease in these biomarkers according to the drug dose has also changed in the same direction. The data from the present study suggest that TFF2 and HYP may be used as early biomarkers for gastric mucosal damage caused by NSAIDs in sheep. However, further studies are needed to clarify the limitations of these biomarkers by using other measurement methods in tissue and plasma.

NSAIDs may impair renal autoregulation under conditions of hypovolaemia and hypotension. It has been reported that a decrease in renal blood flow and function may lead to acute renal failure and death in dogs and cats (Lascelles et al., 2007). It has also been reported that patients who were administered a high daily dose of NSAIDs showed a greater risk for AKI than those who were administered a low to medium dose (Huerta et al., 2005). In a gentamicin-induced renal injury model, Luo et al. (2016) determined that NGAL and KIM-1 gene expressions were the earliest responses of the kidney damage. Alterations in the concentrations of KIM-1 and NGAL had been detected even before significant changes were observed in other clinical chemistry parameters. In the present study, an increase in the concentrations of KIM-1 and NGAL was observed within $3 \mathrm{~h}$. The increase in KIM-1 and NGAL concentrations in G8 was seen as early as after $3 \mathrm{~h}$, before any changes occurred in the conventional parameters $(\mathrm{Cr}$ and BUN) in this group (Table 1). This indicates that NGAL and KIM-1 reflect renal damage early and accurately (Vaidya et al., 2010; Luo et al., 2016). In addition, the level of Cr increased with NGAL and KIM-1 at the same time and this may indicate serious damage to the kidneys at a dose of $16 \mathrm{mg} / \mathrm{kg}$ of TA. The use of high doses of NSAIDs daily is more likely to cause renal injury (Huerta et al., 2005). Further, alterations in the concentration of NGAL were one of the earliest effects observed in the kidneys of animals treated with the $4 \mathrm{mg} / \mathrm{kg}$ dose of TA (G4) after $9 \mathrm{~h}$. However, the earliest effects in KIM-1 in G4 were observed only after $18 \mathrm{~h}$. A similar elevation in the concentrations of NGAL (295\%) and KIM-1 $(726 \%)$ with the levels of creatinine $(363 \%)$ was seen in G16. The increases in renal parameters including NGAL (220\%) and KIM-1 (530\%) were more evident in the G8 group, but this increase was just $5 \%$ in a conventional parameter such as creatinine. These data may suggest that NGAL is an earlier marker of renal injury than KIM-1. The results obtained in a previous study (Noyan et al., 2015) support the use of urinary NGAL concentrations rather than urinary KIM-1 as an early marker for renal hydronephrosis. However, this study had some limitations such as the examination of microalbuminuria and the low number of animals in each group. 
Table 2

Check-up profile of the groups at baseline level (mean $\pm \mathrm{SE})$

\begin{tabular}{llllcccc}
\hline Groups & $\begin{array}{c}\text { Creatinine } \\
(\mu \mathrm{mol} / \mathrm{L})\end{array}$ & $\begin{array}{c}\text { Urea } \\
(\mathrm{mmol} / \mathrm{mL})\end{array}$ & $\begin{array}{c}\text { ALP } \\
(\mathrm{U} / \mathrm{L})\end{array}$ & $\begin{array}{c}\text { ALT } \\
(\mathrm{U} / \mathrm{L})\end{array}$ & $\begin{array}{c}\text { AST } \\
(\mathrm{U} / \mathrm{L})\end{array}$ & $\begin{array}{c}\text { GGT } \\
(\mathrm{U} / \mathrm{L})\end{array}$ & $\begin{array}{c}\text { Total protein } \\
(\mathrm{g} / \mathrm{L})\end{array}$ \\
\hline G0 & $45 \pm 1.5$ & $7 \pm 0.2$ & $85 \pm 16$ & $12 \pm 1.4$ & $91 \pm 13$ & $46 \pm 5.5$ & $69 \pm 1$ \\
G2 & $47 \pm 2.6$ & $7 \pm 0.7$ & $87 \pm 12$ & $13 \pm 2.7$ & $89 \pm 19$ & $44 \pm 4.6$ & $69 \pm 2.9$ \\
G4 & $49 \pm 3$ & $6 \pm 0.5$ & $78 \pm 8$ & $13 \pm 1.2$ & $78 \pm 14$ & $44 \pm 6.2$ & $70 \pm 1.4$ \\
G8 & $47 \pm 3.1$ & $7 \pm 0.4$ & $89 \pm 8$ & $14 \pm 2.8$ & $95 \pm 18$ & $46 \pm 4.4$ & $70 \pm 2.1$ \\
G16 & $46 \pm 3.2$ & $6 \pm 0.2$ & $92 \pm 11$ & $17 \pm 3.2$ & $91 \pm 21$ & $41 \pm 2.7$ & $75 \pm 2.8$ \\
\hline
\end{tabular}

The effects of NSAIDs on the coronary vasculature can be ascertained by the relative inhibition of the COX enzymes (COX-1 and COX-2). These enzymes show counterproductive effects on the coronary vasculature (Cheetham et al., 2008). In vitro data support that TA has a COX-1-sparing (COX-2-selective) activity (Budsberg, 2009). In the present study, the role of widely applied biomarkers such as cTn-I and CK-MB in the blood plasma was evaluated in the detection of cardiac injury. The cTn-I level in G16 was statistically higher than that in G0, G2, G4, and G8 after 9 and 36 h. CK-MB activity in G16 was found to be statistically higher than that in G0 and G2 after $18 \mathrm{~h}$ and in G0, G2, G4 and G8 after $36 \mathrm{~h}$. Biomarkers such as cTn-I and CK-MB may be used to determine the cardiac damage at a high dose of NSAIDs such as TA $(16 \mathrm{mg} / \mathrm{kg})$ in sheep experimental study models.

In conclusion, the changes in the concentrations of novel biomarkers (TFF2, HYP, NGAL, and KIM-1) in the blood plasma might be used for the early diagnosis of gastric and renal damage in sheep. However, further studies are required to prove the applicability of these biomarkers for diagnosing other tissue damages than TA-related ones.

\section{Acknowledgement}

Preliminary results of this study were presented at the 'Mediterranean Natural Sciences and Engineering Congress' (MENSEC 2017), Montenegro.

\section{References}

Alison, M. R., Chinery, R., Poulsom, R., Ashwood, P., Longcroft, J. M. and Wright, N. A. (1995): Experimental ulceration leads to sequential expression of spasmolytic polypeptide, intestinal trefoil factor, epidermal growth factor and transforming growth factor alpha mRNAs in rat stomach. J. Pathol. 175, 405-414.

Budsberg, S. C. (2009): Non-steroidal anti-inflammatory drugs. In: Gaynor, J. S. and Muir, W. W. (eds) Handbook of Veterinary Pain Management. 2nd edition. Mosby Elsevier, St. Louis, MO. pp. 183-209. 
Cheetham, T. C., Graham, D. J., Campen, D., Hui, R., Spence, M., Levy, G. and Shoor, S. (2008): Myocardial infarction and its association with the use of nonselective NSAIDs: a nested case-control and time-to-event analysis. Perm. J. 12, 16-22.

Curry, S. L., Cogar, S. M. and Cook, J. L. (2005): Nonsteroidal anti-inflammatory drugs: a review. J Am. Anim. Hosp. Assoc. 41, 298-309.

CVMP (1997): Tolfenamic acid summary report. EMEA/MRL/183/97. http://www.ema.europa.eu/ docs/en GB/document library/Maximum Residue Limits_Report/2009/11/WC5000156 20.pdf (accessed 25 April 2018).

Diniz, M. S., Teixeira-Neto, F. J., Celeita-Rodríguez, N., Girotto, C. H., Fonseca, M. W., OliveiraGarcia, A. C. and López-Castañeda, B. (2018): Effects of 6\% tetrastarch and lactated Ringer's solution on extravascular lung water and markers of acute renal injury in hemorrhaged, isoflurane-anesthetized healthy dogs. J. Vet. Intern. Med. 32, 712-721.

Farrell, J. J., Taupin, D., Koh, T. J., Chen, D., Zhao, C. M., Podolsky, D. K. and Wang, T. C. (2002): TFF2/SP-deficient mice show decreased gastric proliferation, increased acid secretion, and increased susceptibility to NSAID injury. J. Clin. Invest. 109, 193-204.

Fuchs, T. C. and Hewitt, P. (2011): Biomarkers for drug-induced renal damage and nephrotoxicity - an overview for applied toxicology. AAPS J. 13, 615-631.

Hermann, M. (2009): Cardiovascular risk associated with nonsteroidal anti-inflammatory drugs. Curr. Rheumatol. Rep. 11, 31-35.

Huerta, C., Castellsague, J., Varas-Lorenzo, C. and García Rodríguez, L. A. (2005): Nonsteroidal anti-inflammatory drugs and risk of ARF in the general population. Am. J. Kidney Dis. 45, 531-539.

Kaise, M., Niwa, J., Fujimoto, A., Tashiro, J., Tagami, D., Sano, H. and Ohmoto, Y. (2013): Influence of Helicobacter pylori status and eradication on the serum levels of trefoil factors and pepsinogen test: serum trefoil factor 3 is a stable biomarker. Gastric Cancer 16, 329-337.

Lascelles, B. D., Court, M. H., Hardie, E. M. and Robertson, S. A. (2007): Nonsteroidal antiinflammatory drugs in cats: a review. Vet. Anaesth. Analg. 34, 228-250.

Luo, Q. H., Chen, M. L., Chen, Z. L., Huang, C., Cheng, A. C., Fang, J., Tang, L. and Geng, Y. (2016): Evaluation of KIM-1 and NGAL as early indicators for assessment of gentamycininduced nephrotoxicity in vivo and in vitro. Kidney Blood Press Res. 41, 911-918.

Noyan, A., Parmaksiz, G., Dursun, H., Ezer, S. S., Anarat, R. and Cengiz, N. (2015): Urinary NGAL, KIM-1 and L-FABP concentrations in antenatal hydronephrosis. J. Pediatric Urol. 11, 249. e1-6.

Ozer, J. S., Dieterle, F., Troth, S., Perentes, E., Cordier, A., Verdes, P., Staedtler, F., Mahl, A., Grenet, O., Roth, D. R., Wahl, D., Legay, F., Holder, D., Erdos, Z., Vlasakova, K., Jin, H., Yu, Y., Muniappa, N., Forest, T., Clouse, H. K., Reynolds, S., Bailey, W. J., Thudium, D. T., Topper, M. J., Skopek, T. R., Sina, J. F., Glaab, W. E., Vonderscher, J., Maurer, G., Chibout, S. D., Sistare, F. D. and Gerhold, D. L. (2010): A panel of urinary biomarkers to monitor reversibility of renal injury and a serum marker with improved potential to assess renal function. Nat. Biotechnol. 28, 486-494.

Poulsen, S. S., Thulesen, J., Christensen, L., Nexø, E. and Thim, L. (1999): Metabolism of oral trefoil factor 2 (TFF2) and the effect of oral and parenteral TFF2 on gastric and duodenal ulcer healing in the rat. Gut 45, 516-522.

Shao, X., Li, M., Luo, C., Wang, Y. Y., Lu, Y. Y., Feng, S., Li, H., Lang, X. B., Wang, Y. C., Lin, C., Shen, X. J., Zhou, Q., Jiang, H. and Chen, J. H. (2015): Effects of rapamycin against paraquat-induced pulmonary fibrosis in mice. J. Zhejiang Univ. Sci. B 16, 52-61.

Sistare, F. D., Dieterle, F., Troth, S., Holder, D. J., Gerhold, D., Andrews-Cleavenger, D., Baer, W., Betton, G., Bounous, D., Carl, K., Collins, N., Goering, P., Goodsaid, F., Gu, Y. Z., Guilpin, V., Harpur, E., Hassan, A., Jacobson-Kram, D., Kasper, P., Laurie, D., Lima, B. S., Maciulaitis, R., Mattes, W., Maurer, G., Obert, L. A., Ozer, J., Papaluca-Amati, M., Phillips, J. A., Pinches, M., Schipper, M. J., Thompson, K. L., Vamvakas, S., Vidal, J. M., 
Vonderscher, J., Walker, E., Webb, C. and Yu, Y. (2010): Towards consensus practices to qualify safety biomarkers for use in early drug development. Nat. Biotechnol. 28, 446-454.

Takeuchi, K., Ohishi, M., Endo, K., Suzumura, K., Naraoka, H., Ohata, T., Seki, J., Miyamae, Y., Honma, M. and Soga, T. (2014): Hydroxyproline, a serum biomarker candidate for gastric ulcer in rats: a comparison study of metabolic analysis of gastric ulcer models induced by ethanol, stress, and aspirin. Biomark. Insights 9, 61-66.

Takeuchi, K., Ohishi, M., Ota, S., Suzumura, K., Naraoka, H., Ohata, T., Seki, J., Miyamae, Y., Honma, M. and Soga, T. (2013): Metabolic profiling to identify potential serum biomarkers for gastric ulceration induced by nonsteroid anti-inflammatory drugs. J. Proteome Res. 12, 1399-1407.

Undhad, V. V., Fefar, D. T., Jivani, B. M., Gupta, H., Ghodasara, D. J., Joshi, B. P. and Prajapati, K. S. (2012): Cardiac troponin: an emerging cardiac biomarker in animal health. Vet. World 5, 508-511.

Vaidya, V. S, Ozer, J. S., Dieterle, F., Collings, F. B., Ramirez, V., Troth, S., Muniappa, N., Thudium, D., Gerhold, D., Holder, D. J., Bobadilla, N. A., Marrer, E., Perentes, E., Cordier, A., Vonderscher, J., Maurer, G., Goering, P. L., Sistare, F. D. and Bonventre, J. V. (2010): Kidney injury molecule-1 outperforms traditional biomarkers of kidney injury in preclinical biomarker qualification studies. Nat. Biotechnol. 28, 478-485.

Wallace, J. L. (2008): Prostaglandins, NSAIDs, and gastric mucosal protection: why doesn't the stomach digest itself. Physiol. Rev. 88, 1547.

Zhang, W., Liu, K., Li, L., Li, Y., Sui, X., Rao, Y., Wu, J. and Wu, Q. (2016): Therapeutic effect of low molecular weight chitosan containing sepia ink on ethanol-induced gastric ulcer in rats. Acta Bras. Cir. 31, 813-820.

Zhao, C., Ozaeta, P., Fishpaugh, J., Rupprecht, K., Workman, R., Grenier, F. and Ramsay, C. (2010): Structural characterization of glycoprotein NGAL, an early predictive biomarker for acute kidney injury. Carbohydr. Res. 345, 2252-2261. 